\title{
The Design of Mobile Application Software in Vehicle Monitoring System Based on 3G Intelligence Mobile Phone
}

\author{
Hao $\mathrm{Li}^{1}$, Jie Yang ${ }^{2}$ and Junan $\mathrm{Di}^{3^{*}}$ \\ ${ }^{1}$ Straits Institute, Minjiang University, Fuzhou, Fujian 350121, P.R. China \\ ${ }^{2}$ School of Information Engineering, Wuhan University of Technology, Wuhan 430070, P.R. China \\ ${ }^{3}$ Department of Electronic Information Engineering, Minjiang University, Fuzhou 350121, P.R. China
}

\begin{abstract}
With the popularity of $3 \mathrm{G}$ services, the smart phone performance and user groups continue to rise. The mobile application service capabilities have been expanded to the terminal. It is the inevitable trend for the development of social information to provide information services for users at anytime and anywhere. Based on the mobile application software design of the vehicle monitoring system of $3 \mathrm{G}$ smart phone, this paper has provided a flexible and efficient solution to the vehicle monitoring supportive decision. Monitoring systems of the similar kind have been widely used in all industries.
\end{abstract}

Keywords: Mobile application, vehicle monitoring, software design.

\section{INTRODUCTION}

With the $3 \mathrm{G}$ business in full swing in China, the performance of smart phones and user groups have been the rising power, expanding the strong application service capabilities of the Internet to the terminal. It is an inevitable trend of the development of social information to provide the users with information services at anytime and anywhere. GIS technology has been changed from the traditional standalone applications to Internet-based applications. With the rapid development of the smart phone, smart phone platform will become the new application platform of the GIS technology Internet application.

\section{ANDROID SYSTEMS}

Android is a Linux kernel based operating system. It is the mobile operating system announced on November 5th, 2007, which is earlier developed by Google. It is developed then by the Open Handset Alliance. It uses the architecture of software STack, also known as the architecture of the software stack and is mainly divided into three parts. The underlying Linux kernel only provides the basic functions and the other application software is developed by the companies themselves, with part of the program written in Java. The Android SDK has currently released version 2.2. Compared with the previous version, regardless of function or application execution speed, it has greatly improved and enhanced.

\section{THE OVERALL STRUCTURE OF THE VEHICLE MONITORING MANAGEMENT PLATFORM}

The vehicle monitoring system is composed of by four parts, the vehicle terminal, data center, the control terminal and the map server. The vehicle terminal passes the collected GPS location information and vehicle status information to the data center through the GPRS network. In addition, the data center saves the information and passes forward to the monitoring terminal. When the monitoring terminal has obtained the information, combined with the map information provided by the map server, it displays intuitively the current state of the monitoring of vehicles, and issues instructions to the vehicle terminal. (See Fig. (1))

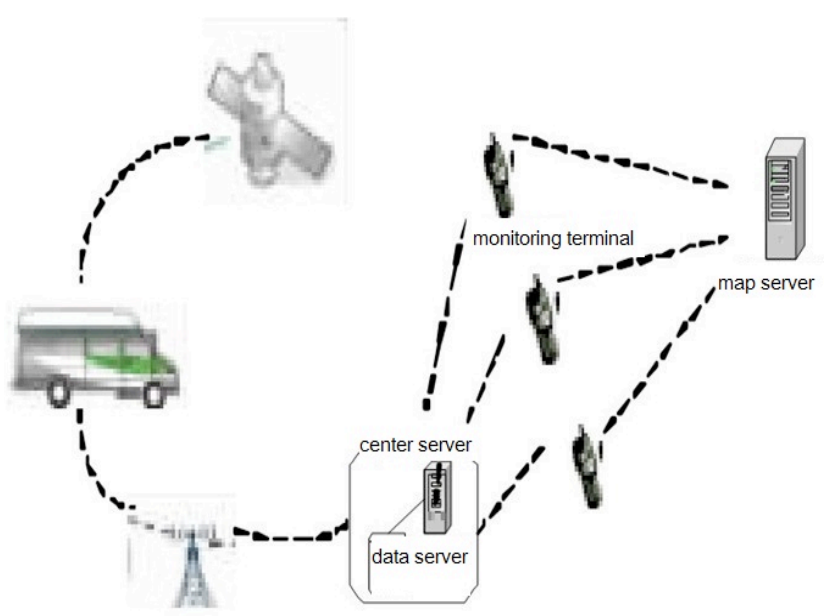

Fig. (1). Vehicle monitoring and management system architecture.

\subsection{The Vehicle Terminal}

Vehicle terminal is composed of by the GPS signal receiver module, the data processing module and GPRS modules. GPS signal module mainly receives GPS signals coming from positioning satellites. Data processing module captures the GPS signal of the GPS signal module and sends GPS location data to background monitoring center 
through the GPRS module so as to realize the real-time monitoring.

\subsection{Data Center}

The data center includes two parts: the central database and the data communications services. The central database stores the GPS data and other data. The data communication services are based on the $\mathrm{C} / \mathrm{S}$ mode, of which the main function is to maintain the vehicle terminal, the connection of monitoring terminals and the data center, and assume the exchange bridge between the vehicle terminal data and monitoring the terminal data.

\subsection{Monitoring Terminal}

Monitoring terminal is to use smart phones (Android smart phones) and connect the monitoring and management system and data center servers. It receives the vehicle terminal and passes forward the data and issues control instructions. Monitoring and management platform is the application program of the $\mathrm{C} / \mathrm{S}$ mode. It provides users with a mapbased monitoring and management platform, including the vehicle back office management and vehicle monitoring system. The main functions of the vehicle monitoring system include electronic map service, real-time monitoring of vehicles tracking, vehicle history trajectory playback, vehicle fences, and alarm processing module. The specific design is seen in the section 4 .

\subsection{Map Server}

Map Server refers to the map server provided by Google Corporation. Monitoring terminal uses the Google API plugin comes from the Google Android system and gets directly access the map server in order to provide an electronic map needed by the monitoring users. It provides an intuitive visualization of geographic information for the user's monitor.

\section{ANDROID-BASED MONITORING AND MAN- AGEMENT SYSTEM DESIGN}

Android-based monitoring and management includes mainly the monitoring and management. Management is the management of vehicle-related information, including drivers' basic information, vehicle information, car records, and alarm records. Monitoring is based on the electronic map to show the way to monitor the vehicle, the features of which include real-time tracking of vehicle location, vehicle history track playback, vehicle fence, and the alarm information processing. The following main key points are to discuss the map-based vehicle monitoring design.

\subsection{Google API Plug-In}

Google API plug-in is an extension of the Android SDK development environment. Through the plug-in, Android application program can easily access Google services and data. The core functionality of the plug-in is the Maps external library, through which its powerful map functionality can be added to the Android application.

\subsection{The Real-Time Vehicle Monitoring}

Users can realize the real-time monitoring of vehicle location on the electronic map. This system adjusts the Map server raster image data and gives it map display Through the Google API plug in Android. (See Fig. (2)) Users want to realize the real-time monitoring of vehicles. In addition, they would like to display the vehicle location information above the map. The first thing to do is for the users to select the vehicle and initiate real-time monitoring requests to the server. When the central server has received the location information sent back from the vehicle terminal, it will pass forward to the monitoring client. After getting the location information, get the current location map and present to the user through the Google API plug-in. The whole process is the collaborative work of the central server and the Google Maps server, and efficient integration of distributed network resources.

\subsection{The Historical Track Playback}

The historical trajectory back reproduces the moving vehicle in a period of time. It has shown on the map with the form of points and lines. The monitoring system needs first to initiate a request to the central server and ask historical data on certain cars for a certain period of time. When the

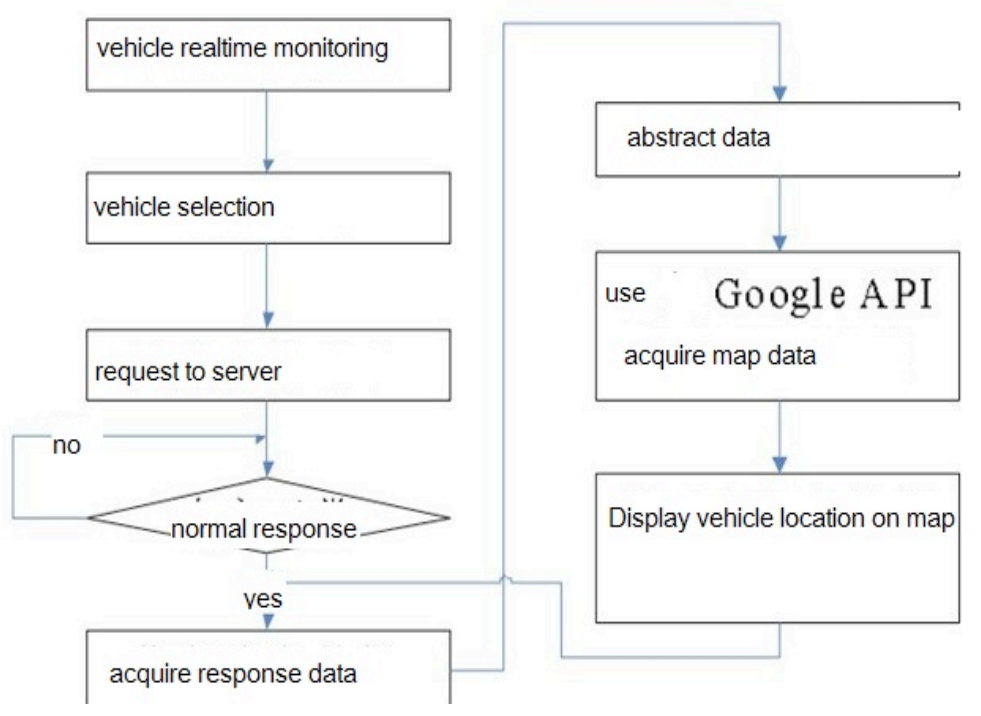

Fig. (2). Vehicle real-time monitoring process. 
central server receives the request, it inquires the history data of the vehicle from the database in this period of time and sends to the monitoring terminal. Monitor terminal calls the plug-in to obtain the map information display. Here, the historical data sent back from the central server may not be the all the data of the car terminal in the period of time. Within a certain period of time of requests, the vehicle may stay in a particular location or in a smaller range of activities. There is no need to display this data on the monitoring terminal at this time. These points are simply displayed as a location so as to avoid the historical track playback. When the vehicle terminal stays in a location on the electronic map for too long, it impresses the users with the illusion of the "crash", and improve the intuitive feelings of the users. In addition, it has reduced the transmission of data between central server and monitoring client.

It is noteworthy that when historical data is too much, the monitoring terminal requires a longer transmission time. In order to avoid excessive waiting of the users, the central data server sends the data sub-batches to monitor terminal, which can receive data and display data at the same time. Adopt the asynchronous manner so as to enhance the working efficiency of the system.

\section{CONCLUSION}

Based on the Android smart phone, the design of the vehicle monitoring management system has been realized. This paper has provided a flexible and efficient solution to the vehicle monitoring supportive decision. Monitoring systems of the similar kind have been widely used in all industries. With the in-depth application and integrated technology development, it has even greater impact on people's lives.

\section{CONFLICT OF INTEREST}

The authors confirm that this article content has no conflict of interest.

\section{ACKNOWLEDGEMENTS}

This paper is the subsidy project of national science foundation key project (project authorization number50830203). I would like to express my gratitude here!

Foundation program: project supported by the State Key Program of National Natural Science of China (Grant No. 51479159); The Education Department of Fujian province science and technology research project (JB12155); Fujian branch of research project of National Occupation Education Research Institute (GZM13012); Project supported by special fund of central government financial support for the development of local colleges and universities (Min Cai -JiaoZhi-2014-50).

\section{REFERENCES}

[1] Z. L. Liu, "Environmental Kuznets Curve: A New Computational Test and Modeling”, IJACT, vol. 5, no. 5, pp. 551-558, 2013.

[2] B. A. Franck and Y. Watanabe, "Security Levels in Countries for International Terminal Operations", Advances in Industrial Engineering and Management, vol. 3, no. 4, pp. 1-8, 2014.

[3] J. Wang, Z. L. Liu, and Y. Chen, "Comparative Analysis of Economic Efficiency of Grain Storage by Solar Absorption Refrigeration", AISS: Advances in Information Sciences and Service Sciences, vol. 4, no. 14, pp. 341-348, 2012. doi: 10.4156/AISS.vol4.issue 14.42

[4] Z. L. Liu, "The Dynamic Analysis of China's Energy-EconomyEnvironment System: VAR and VEC Modeling”, Advances in Information Sciences and Service Sciences, vol. 4, no. 14, pp. 210$218,2012$.

[5] D. Bhattacharya and S. Roychowdhury, "A Constrained Cost Minimizing Redundancy Allocation Problem in Coherent Systems with Non-overlapping Subsystems", Advances in Industrial Engineering and Management, vol. 3 no. 3, pp. 1-6, 2014.

Received: September 18, 2014

Revised: December 22, 2014

Accepted: December 31, 2014

(C) Di et al.; Licensee Bentham Open.

This is an open access article licensed under the terms of the Creative Commons Attribution Non-Commercial License (http://creativecommons.org/licenses/by$\mathrm{nc} / 3.0 /$ ) which permits unrestricted, non-commercial use, distribution and reproduction in any medium, provided the work is properly cited. 